\title{
Catalytic Gels for a Prebiotically Relevant Asymmetric Aldol Reaction in Water: From Organocatalyst Design to Hydrogel Discovery and Back Again
}

\author{
Kirsten Hawkins, Anna K. Patterson, Paul A. Clarke,* and David K. Smith* \\ Cite This: J. Am. Chem. Soc. 2020, 142, 4379-4389 \\ Read Online
}

ACCESS

|lll Metrics \& More | 回 Article Recommendations

S1 Supporting Information

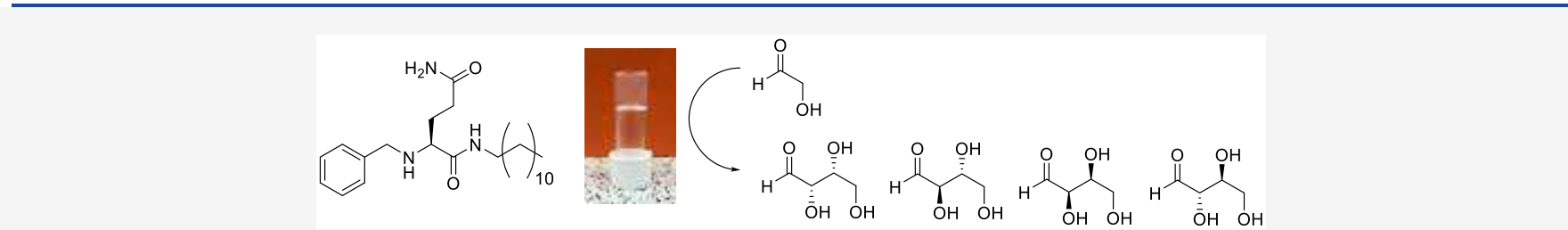

ABSTRACT: This paper reports an investigation into organocatalytic hydrogels as prebiotically relevant systems. Gels are interesting prebiotic reaction media, combining heterogeneous and homogeneous characteristics with a structurally organized active "solid-like" catalyst separated from the surrounding environment, yet in intimate contact with the solution phase and readily accessible via "liquid-like" diffusion. A simple self-assembling glutamine amide derivative $\mathbf{1}$ was initially found to catalyze a model aldol reaction between cyclohexanone and 4-nitrobenzaldehyde, but it did not maintain its gel structure during reaction. In this study, it was observed that compound 1 could react directly with the benzaldehyde to form a hydrogel in situ based on Schiff base 2 as a low-molecular-weight gelator (LMWG). This new dynamic gel is a rare example of a two-component self-assembled LMWG hydrogel and was fully characterized. It was demonstrated that glutamine amide $\mathbf{1}$ could select an optimal aldehyde component and preferentially assemble from mixtures. In the hunt for an organocatalyst, reductive conditions were applied to the Schiff base to yield secondary amine 3, which is also a highly effective hydrogelator at very low loadings with a high degree of nanoscale order. Most importantly, the hydrogel based on 3 catalyzed the prebiotically relevant aldol dimerization of glycolaldehyde to give threose and erythrose. In buffered conditions, this reaction gave excellent conversions, good diastereoselectivity, and some enantioselectivity. Catalysis using the hydrogel of 3 was much better than that using non-assembled 3-demonstrating a clear benefit of self-assembly. The results suggest that hydrogels offer a potential strategy by which prebiotic reactions can be promoted using simple, prebiotically plausible LMWGs that can selectively self-organize from complex mixtures. Such processes may have been of prebiotic importance.

\section{INTRODUCTION}

Gels are a colloidal state of matter in which a solid-like network is dispersed through a continuous liquid-like phase, leading to solvent immobilization. ${ }^{1}$ In the case of supramolecular gels, low-molecular-weight gelators (LMWGs) selfassemble via intermolecular non-covalent interactions into a nanoscale sample-spanning "solid-like" network. ${ }^{2}$ Hydrogels have wide-ranging potential applications, particularly as biomaterials $^{3}$ and in environmental remediation. ${ }^{4}$ In some cases, gelation requires two distinct chemical components ${ }^{5}-$ such "two-component gels" are tunable but relatively rare, especially in the case of hydrogels that self-assemble from LMWGs in water. Examples include systems in which the two components form a non-covalent complex that acts as the LMWG, ${ }^{6}$ or in which they come together to form the LMWG in a dynamic reaction, such as acylhydrazone formation. There are also examples of small molecules that undergo dynamic reactions, like imine formation, to form polymer network hydrogels, ${ }^{8}$ but such gels are more like polymer gels ${ }^{9}$ than those that self-assemble from LMWGs via non-covalent interactions. There has been interest in the development of gels for applications in catalysis. ${ }^{10}$ Gels benefit from a combination of heterogeneous and homogeneous characteristics, allowing the rapid diffusion of small molecules, like reagents and products, through the gel matrix while potentially immobilizing the catalyst by incorporation into, or interaction with, the gel nanofibers. Self-assembled organocatalytic gels have been developed, ${ }^{11}$ with a particular focus on systems in which catalysis is enhanced compared with the non-assembled organocatalyst. In landmark work, Escuder, Miravet, and coworkers demonstrated the potential of gel networks to achieve a range of catalytic processes, including amino acid-mediated aldol reactions. ${ }^{12}$ They also developed self-sorted gel networks with orthogonal catalytic sites to achieve multi-step reactions in one system, demonstrating the potential of gels to act as systems for complex processes. ${ }^{13}$

Received: December 6, 2019

Published: February 5, 2020 
Scheme 1. Outline Synthesis of the New Gels Reported Here and Summary of Their Ability to Catalyze Aldol Reactions

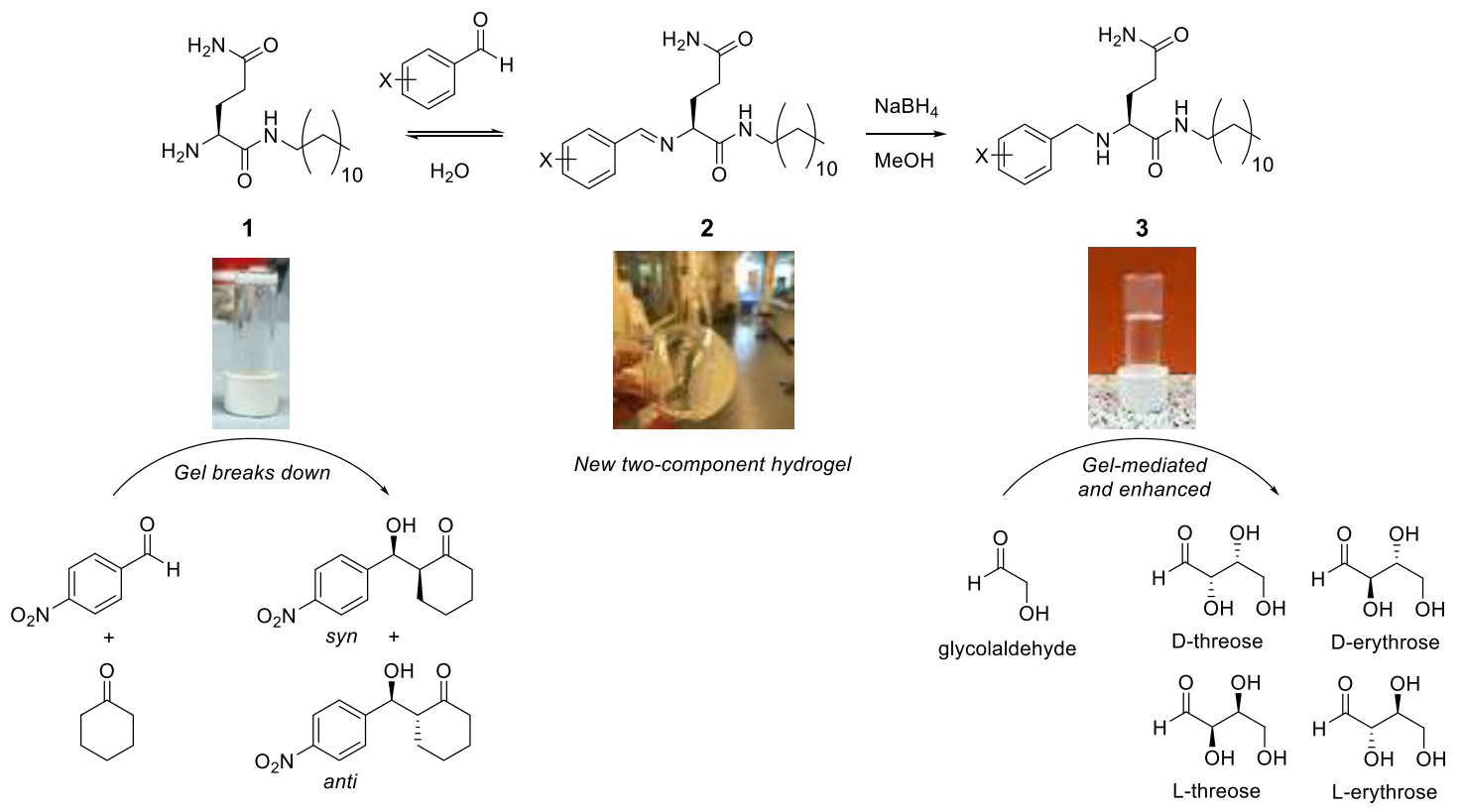

Organocatalysis also plays a key role in the field of prebiotic chemistry, in which researchers aim to demonstrate mechanisms by which simple chemical building blocks, such as those present on the early Earth, can be converted into the more complex chemical constituents of life. ${ }^{14}$ It is hypothesized that some of the dominant reactions of prebiotic chemistry are aldol condensations, which can result in the synthesis of sugars and complex carbohydrates. ${ }^{15}$ It has been argued that the first sugars arose from the formose reaction-an autocatalytic reaction of formaldehyde to form glycolaldehyde, ${ }^{16}$ followed by aldol homologation of glycoladehyde into higher sugars. Alternatively, it has been suggested by Sutherland that glycolaldehyde and higher sugars can be generated from formaldehyde by iterative Kiliani-Fischer synthesis and aminooxazoline formation. ${ }^{17}$

It was first reported that prebiotically relevant amino acids, such as proline, could catalyze asymmetric aldol cyclizations in the early 1970s. ${ }^{18}$ In 2000, Barbas and List studied proline and other primary and secondary, cyclic and acyclic amino acids as general aldol catalysts, proposing a six-membered enamine Zimmerman-Traxler transition state. ${ }^{19}$ In key work, Pizarello and Weber showed that the dimerization of glycolaldehyde by $\alpha, \alpha$-L-disubstituted amino acids led to the formation of $\mathrm{L}$ tetroses in $7 \%$ enantiomeric excess (ee). ${ }^{20}$ Similar studies by Breslow with stoichiometric quantities of amino acids showed the formation of D-glyceraldehyde with similar ee's. ${ }^{21}$ Dipeptides have shown improved reaction selectivity and tetrose yield, ${ }^{22}$ and zinc-proline complexes can catalyze an aqueous aldol reaction in which a cocktail of higher carbohydrates was produced. ${ }^{23}$ Barbas demonstrated the catalysis of aldol reactions on activated nitrobenzaldehydes in water without the need for co-solvents by using proline modified with a hydrophobic group. Micellar assembly of the catalyst mediates the reaction by providing a hydrophobic environment for the organic reagents. ${ }^{24}$ This led Janda and coworkers to critically question what was really meant by performing organocatalysis "in water"-which remains a key question in the field. ${ }^{25}$ They also noted that adding an acid to limit general base catalysis enhanced enantioselectivity. Clarke and co-workers went on to apply this approach to prebiotically relevant reactions, reporting the aldol condensation of TIPSprotected glycolaldehyde in water promoted by amino acid esters. $^{26}$ They then reported aldol reactions on unprotected glycoladehyde to generate threose and erythrose, ${ }^{27}$ and demonstrated a plausible prebiotic synthesis in water of 2deoxy-D-ribose using amino acid esters and amino nitriles as organocatalysts. ${ }^{28}$

Although gels have been studied to some extent in the field of organocatalysis as described above, only Escuder and coworkers have performed a prebiotically relevant reaction, using a tripeptide organogelator to catalyze the reaction between TIPS-protected glycolaldehyde, yielding TIPS-protected threose and erythrose derivatives. ${ }^{29}$ In this research, the catalysis failed on the unprotected glycolaldehyde. The relative lack of development of self-assembled prebiotic organocatalytic gels is surprising, given that such systems can form from simple, prebiotically plausible molecular-scale building blocks. Furthermore, gels are known to play vital roles in life itself; for example, the cytoplasm interior of a cell has a gel-like structure. ${ }^{30}$ It has been suggested that gels may have been an effective medium for organization and compartmentalization prior to the evolution of protective membranes. ${ }^{31}$ Gels can, to some extent, control traffic in and out of themselves, and can immobilize larger objects, or interactive ones, effectively separating them from the surrounding environment. We therefore reason that hydrogels are fascinating potential prebiotic materials-the development of gels with prebiotic catalytic potential may provide insight into how gel assembly and catalysis can cooperate in the evolution of more complex yet better organized chemical systems. Indeed, there is considerable current interest in a "systems chemistry" approach to prebiotic chemistry, in which holistic systems of multiple components collaborate in achieving emergent properties ${ }^{32}-$ we suggest self-assembled hydrogels may play an important, and under-recognized, role in such processes. With this in mind, we decided to embark on a program of organocatalyst and hydrogelator discovery with the hope of gaining new insights relevant to both supramolecular gels and prebiotic 
organocatalysis (Scheme 1). We focus here on self-assembled gels as catalysts for a specific prebiotic process-unprotected aqueous glycolaldehyde dimerization-a key step in the pathway leading to sugars.

\section{RESULTS AND DISCUSSION}

Glutamine Derivative 1: Gelation. Preliminary research, in which the behavior of a number of modified amino acids such as compound $\mathbf{1}$ had been screened, ${ }^{33}$ suggested such compounds had the potential to become involved in aldol catalysis. Compound $\mathbf{1}$ was synthesized by coupling dodecylamine and Boc-protected glutamine using 1-ethyl-3-(3dimethylaminopropyl)carbodiimide (EDC) and dimethylaminopyridine (DMAP), followed by acid-mediated removal of the Boc-protecting group (Scheme 2). The product was converted into free base form using $\mathrm{NaOH}(1 \mathrm{M})$ for 2-12 h.

Scheme 2. Synthesis of Glutamine Amide 1

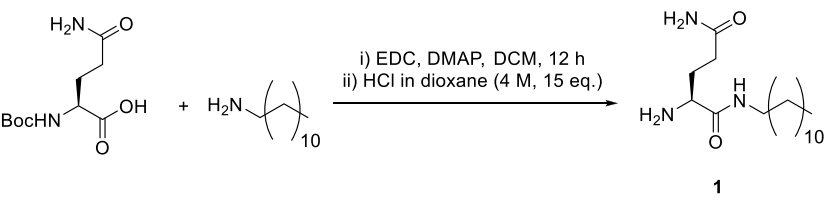

Initially a range of solvents were used to test the gelation of compound $\mathbf{1}$ at $3 \% \mathrm{wt} / \mathrm{vol}$ loading using a simple tube inversion test for gel formation. Partial gels (in which the gel partially prevented dropping of the sample on inversion) were formed in acetonitrile, tetrahydrofuran, and toluene, and a full gel (resistant to tube inversion) was formed in cyclohexane. Most importantly with regard to this study, a gel was formed in water. However, this was somewhat irreproducible, which we attributed to the relatively high solubility of compound $\mathbf{1}$ in water. Rather like in crystallization events, this can make the nucleation of a gel network somewhat temperamental on application of a heat-cool cycle. We therefore did not characterize this gel in detail, but decided instead to move straight on to testing the proficiency of compound $\mathbf{1}$ as an organocatalyst.

Glutamine Derivative 1: Organocatalysis. We tested compound $\mathbf{1}$ in a standard aldol reaction, which proceeds effectively and has easy-to-analyze products-the reaction between cyclohexanone and 4-nitrobenzaldehyde (Scheme 3).

\section{Scheme 3. Aldol Reaction Investigated Using} Organocatalyst 1

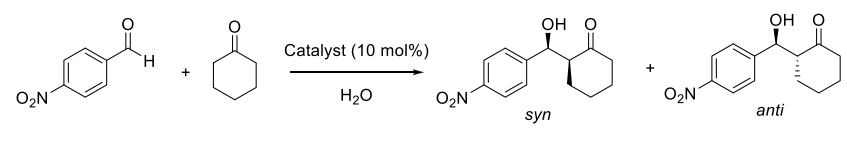

Unfortunately, the gel was not reproducible or stable enough to be used as a catalyst, but to provide some insight into organocatalysis, we probed the ability of compound $\mathbf{1}$ to catalyze this reaction in a heterogeneous "solution" phase.

A solution of 4-nitrobenzaldehyde (1 equiv) in cyclohexanone (10 equiv) was added to a solution of the amide ( 0.1 equiv) in water $(20 \mathrm{~mL})$, and a heterogeneous mixture formed (see Supporting Information (SI), section 5, for full experimental details). Reactions were analyzed after 24,48 , and $72 \mathrm{~h}$-each time point was a separate reaction because the heterogeneous nature of the reaction meant aliquot sampling did not give accurate representation, and each time point was analyzed across triplicate repeat reactions. After the allotted time, the reaction mixture was extracted with dichloromethane (DCM) and solvent removed in vacuo. ${ }^{1} \mathrm{H}$ NMR analysis of the crude product in deuterated chloroform $\left(\mathrm{CDCl}_{3}\right)$ was used to calculate the conversion and diastereomeric ratio (dr) (see SI, Figures S7 and S8 and Table S1).

The reaction proceeded with excellent conversions, up to $98 \%$ after $72 \mathrm{~h}$ with an anti:syn ratio of 1.3:1.0. The anti:syn ratio was higher at shorter reaction times (e.g., 2.1:1.0 at $24 \mathrm{~h}$ ). Chiral HPLC was used to determine the enantioselectivity (SI, Figure S9), with results indicating that the anti product was obtained with an ee of up to $32 \%$, while the syn product was obtained with an ee up to $11 \%$ (SI, Figures S10-S12 and Table S2). However, these ee's varied widely from reaction to reaction, reflective of the highly heterogeneous nature. The relatively low diastereo- and enantioselectivities suggest a relatively poorly organized transition state, as might be expected given just one amino acid is in the organocatalyst. Other studies have shown that dipeptides can enhance selectivity as a result of the introduction of additional noncovalent interactions, ${ }^{22}$ although of course, in prebiotic terms, the catalyst is then more structurally complex, and less likely to spontaneously emerge from simple building blocks. It is also worth noting that co-solvents such as DMSO can be added to homogenize reactions to improve selectivity-for prebiotic relevance we chose to avoid this approach, but in terms of basic process optimization we suggest this would be a useful approach.

Interestingly, on catalysis of the heterogeneous reaction between cyclohexanone and 4-nitrobenzaldehyde mediated by compound 1, we consistently observed that a stable samplespanning gel was formed during the reaction (see Scheme 1). We reasoned that this gel was the result of interactions between the different components in the system, and set out to understand this in more detail.

Schiff Base 2: Two-Component Gelation. To understand gel formation, we tested compound $\mathbf{1}$ on its own and with cyclohexanone and 4-nitrobenzaldehyde individually under the reaction conditions. Only in the presence of 4nitrobenzaldehyde was a hydrogel consistently formed in situ. Mass spectrometry of the gel and analysis of the dried residue by ${ }^{1} \mathrm{H}$ NMR spectroscopy indicated quantitative conversion of catalyst 1 into Schiff base 2 (Scheme 4). There have been

Scheme 4. Reaction between Glutamine Amide 1 and 4Nitrobenzaldehyde to Give Compound 2 Which Assembles into Gels In Situ in Water

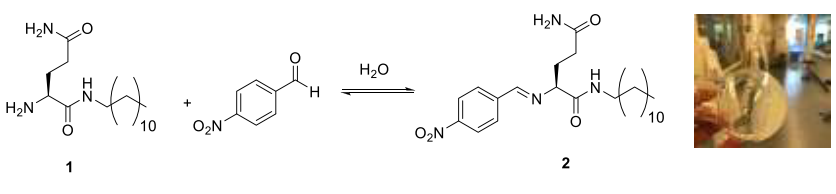

previous reports of two-component gels based on dynamic, reversible imine formation, but in all cases this leads to gels in organic solvents, whereas here the gel is forming in water. ${ }^{34}$ As noted in the Introduction, two-component hydrogels based on LMWGs that self-assemble through non-covalent interactions are relatively rare.

Gel formation was accelerated and optimized by use of a heat-cool cycle to yield highly reproducible gels. These twocomponent gels assembled at minimum gel concentrations 
(MGCs) as low as $0.5 \mathrm{mg} / \mathrm{mL}$ of glutamine amide 1 and 0.24 $\mathrm{mg} / \mathrm{mL}$ ( 1 equiv) of 4-nitrobenzaldehyde, giving a total gelator loading of $0.074 \% \mathrm{wt} / \mathrm{vol}$ (SI, Table S6). This is remarkable performance for a two-component LMWG hydrogel, and allows the system to be categorized as a "supergelator" (i.e., gelation at loadings $<0.1 \% \mathrm{wt} / \mathrm{vol}){ }^{35}$ The maximum gel concentration was a total gelator loading of $0.74 \% \mathrm{wt} / \mathrm{vol}-$ above this level, solubility problems meant that not all of the material dissolved prior to gel formation, and consequently, the resulting gels were not fully homogeneous.

We then characterized these gels in more detail. First, we used simple tube inversion methodology to gain insight into the thermal stability of the gel ( $T_{\text {gel }}$ value) formed from a $1: 1$ mixture of components. As expected, on increasing the concentration, the $T_{\text {gel }}$ increased from $61{ }^{\circ} \mathrm{C}(0.15 \% \mathrm{wt} / \mathrm{vol})$ to $83{ }^{\circ} \mathrm{C}(0.74 \% \mathrm{wt} / \mathrm{vol})$ as the sample-spanning network became more fully established at higher concentration (SI, Table S7).

Obviously, the reaction to form the Schiff base requires 1:1 stoichiometry, but we were interested to know how much aldehyde would be required to assemble a gel. Tests of gelation using $1 \mathrm{mg}$ of glutamine amide 1 indicated that gels would not form with $<0.7$ equiv of 4-nitrobenzaldehyde (SI, Table S5). However, once 0.7 equiv was present, a gel resulted-clearly at this point, sufficient Schiff base is generated to support a sample-spanning gel-phase network (consistent with the MGC of the gelator described above). Gels were still formed by the Schiff base even in the presence of significant excesses of 4nitrobenzaldehyde but, once $>10$ equiv were present the gels became less stable.

Further analysis of the 1:1 gel was performed using ${ }^{1} \mathrm{H}$ NMR spectroscopy. In gel-phase NMR, mobile components can be detected, but the self-assembled "solid-like" network has broadened peaks and is thus not observed. ${ }^{36}$ Spiking the gel with a mobile internal standard allows quantification of any mobile components in the gel. For the 1:1 gel, we did not observe Schiff base 2 in the ${ }^{1} \mathrm{H}$ NMR spectrum, indicating it is indeed assembled into the "solid-like" network (SI, Figure S34). Neither did we observe any mobile unreacted glutamine amide 1. However, we did observe ca. 0.3 equiv of mobile unreacted aldehyde. This suggests, in-line with the observations above, that ca. 0.7 equiv of aldehyde is sufficient to cause the system to assemble into a gel. However, the remaining "excess" unreacted glutamine amide $\mathbf{1}$ is also probably assembled within the gel, as it is not observed as mobile in the NMR.

We then wanted to understand the gelation process. Given that glutamine derivative $\mathbf{1}$ shows some ability to assemble in its own right, and sometimes forms gels, we performed dynamic light scattering (DLS). It was evident based on light scattering that compound $\mathbf{1}$ is indeed self-assembled (SI, Figure S35). It was not possible to provide an accurate size for these assemblies, because DLS can only do this for spherical systems. Interestingly, however, on addition of 4-nitrobenzaldehyde, the emergence of a new peak in the DLS (SI, Figure S36) clearly indicated that the self-assembly mode changes as the two components react with one another, consistent with the formation of a more effective selfassembled gel in situ. This suggests that glutamine amide $\mathbf{1}$ assembles into nanoscale objects, with limited ability to form an interactive $3 \mathrm{D}$ sample-spanning network. However, on reaction with 4-nitrobenzaldehyde, the solubility changesdecreasing in water; this will enhance fiber-fiber interactions, promoting the formation of a sample-spanning network. In this way, the second component drives gelation. This model of selfassembly is reminiscent of the widely explored Fmoc-peptide derivatives-in basic conditions the anionic carboxylate assembles into nanoscale objects (cylindrical micelles), and only on protonation does the lowering of solubility drive fibrillar gel network assembly. ${ }^{37}$

The ability of the isolated and dried Schiff base derivative 2 to form hydrogels directly was also tested. Effective gels could not be directly formed from 2 , probably because the solubility was too low for heating and/or sonication to fully dissolve the system and overcome the energy barrier to gelation. Once again, this is reminiscent of Fmoc peptides, in which the selfassembling carboxylic acid gelator is difficult to assemble into gels directly, and is instead generated at a controlled rate in situ by protonation of the pre-assembled free carboxylate to assemble effective gels. ${ }^{38}$ We argue that, for our new twocomponent Schiff base gelator 2 , the reaction between amine 1 and the aldehyde delivers the LMWG into solution at an appropriate rate for it to then self-assemble into effective gels in situ.

The gels were studied by circular dichroism (CD) spectroscopy to better understand the nanoscale organization of the gelator. The aldehyde component is achiral and has UVactive absorption bands associated with the aromatic ring (ca. $280 \mathrm{~nm}$ ), while the glutamine amide component is chiral, but only has an absorption band at ca. $220 \mathrm{~nm}$ associated with the chiral amide. The results (Figure 1, top) indicated a small CD signal associated with each of the chromophores, demonstrating that, within the gel, both experience a chiral environment; i.e., the achiral aromatic aldehyde is attached to the chiral glutamine amide unit, and thus experiences an induced CD effect. $^{39}$ The CD signal was thermally responsive, as expected for a self-assembled system (Figure 1, bottom). On heating
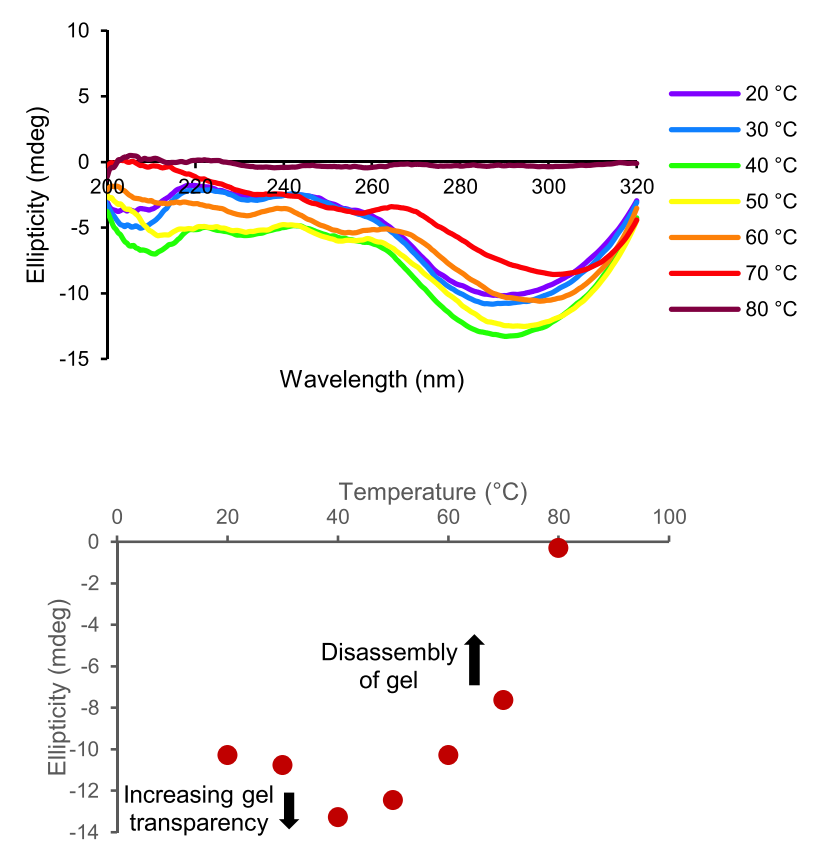

Figure 1. (Top) Circular dichroism (CD) spectra at different temperatures of the gel formed by glutamine amide and 4nitrobenzaldehyde (1:1 ratio) at a total loading of $0.15 \% \mathrm{wt} / \mathrm{vol}$. (Bottom) Thermal response of the ellipticity of the two-component gel at $290 \mathrm{~nm}$. 
from 20 to $40{ }^{\circ} \mathrm{C}$, the $\mathrm{CD}$ signal, somewhat unusually, increased in intensity-a result of the gel becoming slightly more transparent (limiting light scattering). On heating further from 40 to $80{ }^{\circ} \mathrm{C}$, however, the $\mathrm{CD}$ band then decreased in intensity, as would be expected for the thermally induced disassembly of a chiral gel nanostucture. At $80{ }^{\circ} \mathrm{C}$, the system shows no $\mathrm{CD}$ signal, indicative of complete disassembly into isolated molecules of $\mathbf{2}$.

Transmission and scanning electron microscopy (TEM and SEM) provided further insight into the nanoscale morphologies of these gels. Although drying effects can be significant in sample preparation and impact on apparent morphologies, ${ }^{40}$ EM can still be useful for comparing related samples prepared in the same way. We used standard methods to image the gel samples (SI, section 3.1) that avoid any problems associated with ice crystal formation. For SEM imaging (SI, Fig. S42) we applied cryo-drying in an attempt to minimize morphological reorganization during the drying step. The sample with $1 \mathrm{mg} /$ $\mathrm{mL}$ of 1 (total loading ca. $0.15 \% \mathrm{wt} / \mathrm{vol}$ ) had a nanofibrillar morphology as typically observed for self-assembled gels, with nanofiber dimensions estimated by TEM as 55-110 nm (Figure 2 left; SI, Figures S41 and S42). However, on
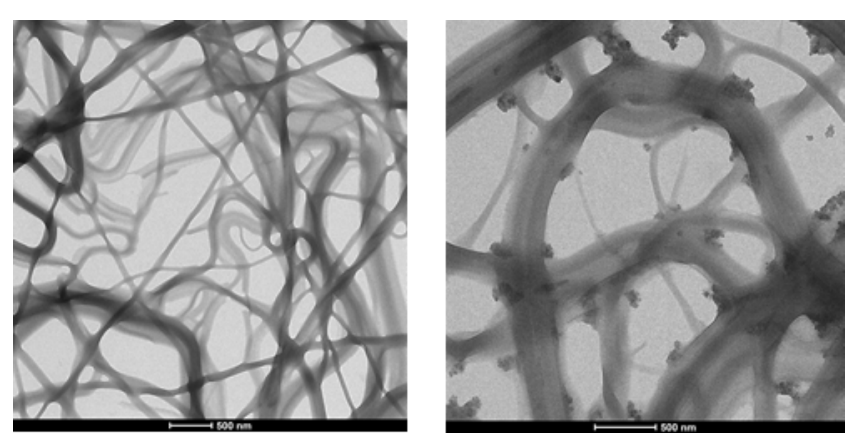

Figure 2. TEM images of dried samples of (left) $1 \mathrm{mg} / \mathrm{mL}$ glutamine amide 1 and $480 \mu \mathrm{g}$ of 4-nitrobenzaldehyde (1:1, loading $0.15 \% \mathrm{wt} /$ vol) and (right) $5 \mathrm{mg} / \mathrm{mL}$ glutamine amide 1 and $2.4 \mathrm{mg}$ of nitrobenzaldehyde ( $1: 1$, loading $0.74 \% \mathrm{wt} / \mathrm{vol})$. Scale bars $=500 \mathrm{~nm}$.

increasing the loading, the fibers became enlarged, and additional nodular aggregates also appeared to be present (Figure 2 right; SI, Figures S43-S45). It seems plausible that as the loading increases, excess material has a secondary aggregation mode. Alternatively, the nodules may be associated with less soluble material as the concentration of gelator is increased.

Rheological studies (see SI) on the combination of glutamine amide $\mathbf{1}$ and 4-nitrobenzaldehyde using parallel plate geometry indicated that the self-assembled materials behave as gels with $G^{\prime}>G^{\prime \prime}$ (SI, Figures S37-S40). Interestingly, the gel exhibited self-healing properties, being broken down by shear during injection and then reforming in situ on standing (SI, Figure S46 and Table S10). This gives this family of gelators potential biomaterials applications-for example in drug delivery. ${ }^{41}$

With our interests in prebiotic chemistry, we then substituted 4-nitrobenzaldehyde for a simpler aldehyde, benzaldehyde, which is known to be a prebiotically plausible building block. ${ }^{42}$ Pleasingly, benzaldehyde behaved in an analogous manner to 4-nitrobenzaldehyde, forming gels. In general, when testing aldehydes (SI, Table S11), we found a preference for aromatic aldehydes over aliphatic aldehydes, but if electron-donating groups were present on the ring, then gelation did not occur. This preference would reflect the inherent electrophilicity of the aldehyde, and the ability of the aromatic ring to self-assemble via $\pi-\pi$ interactions, both preferred in electron-poor systems.

One fascinating property of two-component gels is the potential for one component to select its ideal partner from a mixture of possibilities. ${ }^{43}$ Obviously this has considerable relevance in a prebiotic setting, as it enables order to spontaneously emerge from a relatively complex set of inputs. We therefore tested the assembly of the gel based on glutamine amide 1 in the presence of benzaldehyde (1 equiv) and 4hydroxy-3-methoxybenzaldehyde (vanillin, 1 equiv). Benzaldehyde is capable of supporting gelation (Figure 3, left), but
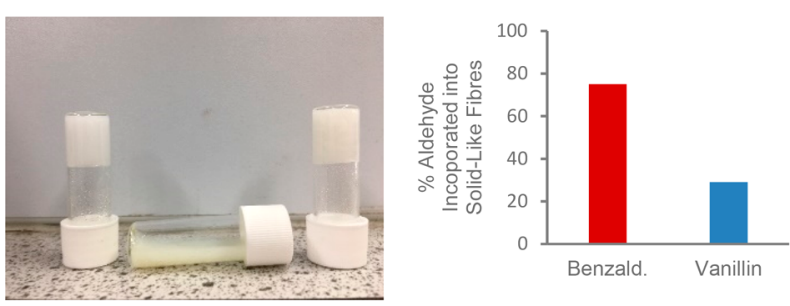

Figure 3. (Left) Photograph of gels formed by (left) 1:1 glutamine amide:benzaldehyde, (middle) 1:1 glutamine amide:vanillin, and (right) 1:1:1 glutamine amide:benzaldehyde:vanillin. (Right) Percentage of benzaldehyde and vanillin incoporated into the solid-like gel nanofibers as assessed by ${ }^{1} \mathrm{H}$ NMR on a gel assembled from 1:1:1 glutamine amide:benzaldehyde:vanillin in $\mathrm{D}_{2} \mathrm{O}$.

vanillin is not (Figure 3, middle). Interestingly, the 1:1:1 glutamine amide:benzaldehyde:vanillin mixed system still formed a gel (Figure 3, right), suggesting benzaldehyde can dominate. We then used ${ }^{1} \mathrm{H}$ NMR analysis ${ }^{43-d}$ on this mixed gel to determine how much of each aldehyde was being incorporated into the "solid-like" gel fibers. This indicated that the gel network incorporated $75 \%$ of the benzaldehyde, but only $29 \%$ of the vanillin (Figure 3; SI, Figures S47 and S48). It is therefore clear that component selection for the preferred aldehyde that gives effective gelation. A majority of the vanillin is left in the solution phase, while a majority of the benzaldehyde becomes assembled into the gel fibers, clearly demonstrating that gel assembly can select specific components from mixtures to assemble functional materials-a principle of prebiotic relevance.

In summary, in the hunt for a catalyst for the aldol reaction between cyclohexanone and 4-nitrobenzaldehyde, we discovered a highly effective two-component hydrogel that forms at low concentrations and can select preferred components from mixtures. Clearly there is very considerable scope for tuning the performance of this class of gels by varying (i) the aldehyde, (ii) the amino acid, or (iii) the hydrophobic chain. Full detailed results of these structure-activity relationship studies will be reported elsewhere.

Secondary Amine 3: Gelation. Having developed a highly effective new two-component hydrogel based on simple building blocks, we then wanted to achieve effective organocatalysis. We reasoned that simple reduction of Schiff base $\mathbf{2}$ would yield a secondary amine that may be an organocatalyst. Furthermore, given that the perturbation to molecular structure is relatively small, we believed it may also form a hydrogel. We opted to use Schiff base $2 \mathbf{a}$, based on benzaldehyde, rather than the Schiff base based on 4- 
nitrobenzaldehyde, as it is simpler and more prebiotically relevant.

To synthesize the Schiff base in high yield, we made use of its gel-forming ability to drive the reaction to completion, giving us a straightforward reaction in water (Scheme 5). We

Scheme 5. Synthesis of Schiff Base 2a from Benzaldehyde and Glutamine Amide 1, Using Gelation to Drive the Reaction to Completion in Water

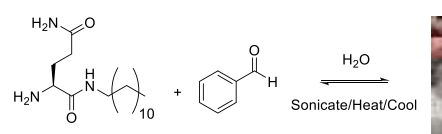

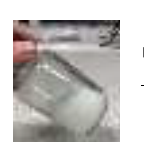

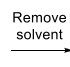

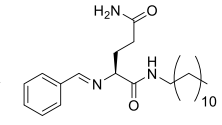

2a suggest that gelation may, in the prebiotic world, have sometimes provided a useful mechanistic driving force for reactions in this way, giving rise to conversion to products and assembly of a discrete environment. Indeed, phase change processes such as crystallization have previously been reported by other researchers to assist in prebiotic processes. ${ }^{44}$ Intriguingly, gelation offers the possibility of the phase change driving the system toward the preferred product, but in a form retaining high solvent porosity and compatibility, giving potential for further interaction/reaction of the selected product with other small molecules.

After drying the gel product $\mathbf{2 a}$, we reduced the imine using sodium borohydride in methanol to give the desired amine 3 in good yield (Scheme 6). There has been interest in reductive

Scheme 6. Reduction of Imine with Sodium Borohydride to Yield Secondary Amine 3

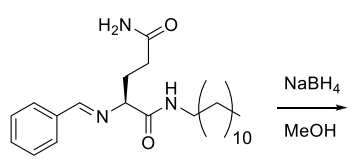

2a

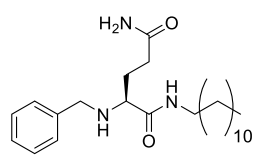

amination, specifically to convert $\alpha$-keto acids into amino acids, with a number of prebiotically plausible approaches discussed in the literature. ${ }^{45}$ As such, we consider this approach to have some prebiotic potential.

We were delighted to find that compound 3 is a highly effective hydrogelator (Figure 3) - even more so than the twocomponent Schiff base. It also formed gels in some organic solvents (SI, Table S12). A heat/sonication/cool cycle was used to achieve consistent and fast (ca. $2 \mathrm{~h}$ ) gel formation, with the MGC being just $0.03 \% \mathrm{wt} /$ vol (SI, Figure S49). This is even lower than the precursor Schiff base, making this a highly potent LMWG. This suggests that the secondary amine may enhance non-covalent interactions between LMWGs.

The $T_{\text {gel }}$ value of this new gelator is $50{ }^{\circ} \mathrm{C}$ at a concentration of $0.10 \% \mathrm{wt} / \mathrm{vol}$. SEM imaging indicates the assembly of a fibrillar sample-spanning network with diameters of ca. $40 \mathrm{~nm}$ (Figure 4). These fibers are smaller than those found in the two-component gel, consistent with the formation of a more stable gel at lower concentrations. It is also consistent with the fact that the visual appearance of the gel is very different from those observed for the two-component hydrogel, being much more optically transparent, as is typical of smaller nanoscale assemblies, which are less able to scatter the incident light.

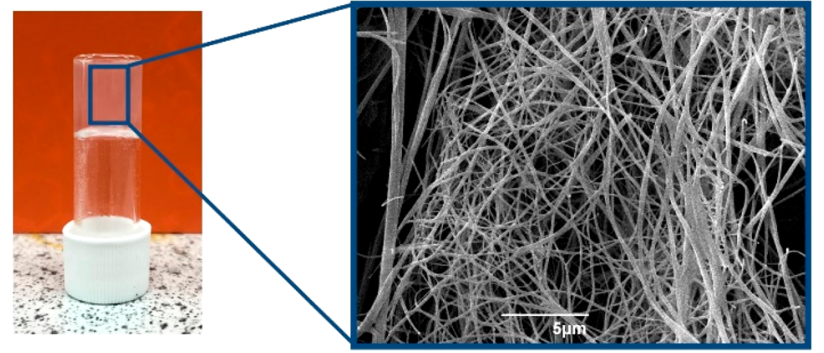

Figure 4. Benzylglutamine amide 3 hydrogel (loading 0.1\% wt/vol) and SEM image of a dried sample of the gel.

The gel was studied by variable-temperature CD spectroscopy (Figure 5). The CD signal was very different from that

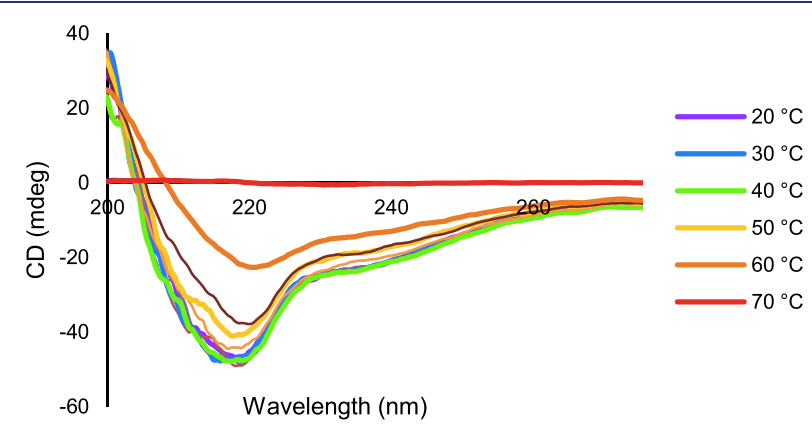

Figure 5. Circular dichroism (CD) spectra at different temperatures of the gel formed by compound $3(0.1 \% \mathrm{wt} / \mathrm{vol})$.

observed for the two-component system, with a much larger band (50 mdeg) at ca. $215 \mathrm{~nm}$, associated with chiral organization of the chiral amide. This compares with much smaller CD bands (ca. 5 mdeg) for the two-component system, and supports the view that secondary amine 3 is a better organized, more effective self-assembling hydrogel. The CD spectrum is thermally responsive; by $70{ }^{\circ} \mathrm{C}$ no $\mathrm{CD}$ signal is observed, confirming it can be attributed to LMWG selfassembly.

Analysis of the gel by ${ }^{1} \mathrm{H}$ NMR spectrosocopy (SI, Figure S50) indicated $100 \%$ incorporation of gelator into the "solidlike" gel network, with no NMR resonances associated with free "mobile" gelator being observed-again consistent with the highly organized assembly of this system.

The rheology of the gel formed by 3 at a loading of $4 \% \mathrm{wt} /$ vol (SI, Figures S51 and S52) indicated an effective soft gel, with $G^{\prime}>G^{\prime \prime}$, and a $G^{\prime}$ value of ca. $200 \mathrm{~Pa}$. The gel crossover point is at ca. $3 \%$ strain. For detailed comparison, we also performed rheology on precursor Schiff base gel 2a, formed from the combination of glutamine amide and benzaldehyde, under exactly the same conditions (SI, Figures S39 and S40). In this case, the gel had a $G^{\prime}$ value of $780 \mathrm{~Pa}$ and a crossover point at ca. $8 \%$ strain. Clearly the precursor gel is somewhat stiffer than the reduced secondary amine version of the gel. This is in-line with its more opaque appearance, indicating a greater degree of crystalline solid-like behavior. Conversely, the secondary amine gelator 3 gives transparent, more nanoscale gels that self-assemble at exceptionally low loadings. We were therefore excited to explore the potential use of these gels in organocatalysis.

Secondary Amine 3: Organocatalysis. Initially, we tested the ability of compound 3 to catalyze the model reaction between cyclohexanone and 4-nitrobenzaldehyde in 
the solution phase, to benchmark the organocatalyst against glutamine amide 1 . In water, the conversion was ca. $43 \%$ after $72 \mathrm{~h}$, and the anti:syn ratio was ca. 2.0:1, with the anti product being produced in a typical ee of $15 \%$ and the syn product in a typical ee of 7\% (SI, Figures S3 and S13-S22). On performing the reaction in buffered conditions at $\mathrm{pH} 7$, although the conversion dropped, the ee's rose to $55 \%$ for the anti product and $17 \%$ for the syn product (SI, Table S4 and Figures S23S25). Once again, we suspect better outcomes could have been achieved by adding a co-solvent, as the system is quite heterogeneous, but reaction optimization was not the goal of our research. On testing this reaction on the gel, once the reagents were applied to the top of the gel, it broke down. We reasoned that the cyclohexanone acts as an organic solvent, dissolving compound 3 and thus disrupting the self-assembled gel network.

We therefore decided to probe the ability of this amine to catalyze a prebiotically relevant aldol reaction that is fully compatible with aqueous-phase reaction conditions-the dimerization of glycolaldehyde (Scheme 7). The gel was

Scheme 7. Dimerization of Glycolaldehyde to Give Threose and Erythrose

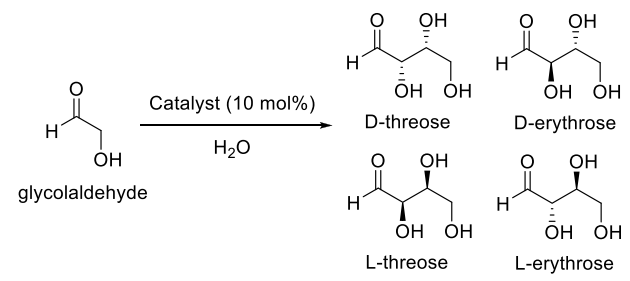

prepared in the standard way $(20 \mathrm{mg}, 49.6 \mu \mathrm{mol}$ in $5 \mathrm{~mL}$ solvent), and the dimer of glycolaldehyde $(59 \mathrm{mg}, 0.49 \mathrm{mmol})$ was dissolved in water $(200 \mu \mathrm{L})$ and then rapidly added $(<1$ min in total $)$ in small aliquots $(20 \times 10 \mu \mathrm{L}$ additions $)$ to the surface of the gel, ensuring dispersion over the gel surface (SI, section 6). Under these conditions, the gel was ca. $\mathrm{pH} 6$ during reaction. The system was monitored for $24 \mathrm{~h}$ to see if the gel remained intact. Initial studies were promising, with the gel being stable even after $48 \mathrm{~h}$.

To analyze the products, a trapping and analysis methodology was developed based on the conversion of the products to diphenyl hydrazone derivatives. Four individual standards were prepared from L- and D-threose and L- and D-erythrose by adding diphenyl hydrazine in methanol with acetic acid and stirring for $1 \mathrm{~h}$ (SI, section 4). ${ }^{33}$ The products were purified by column chromatography and analyzed by chiral HPLC. The four enantiomers could be separated using an IC Chiral-Pak column with hexane:isopropyl alcohol (90:10) at $40{ }^{\circ} \mathrm{C}$ (SI, Figure S26).

Analysis of the dimerization of glycolaldehyde on the catalytic hydrogel was then carried out. At the end of each reaction, the hydrogel was dehydrated in vacuo. To the residue, diphenyl hydrazine in methanol was added, followed by $1-2$ drops of acetic acid, and the mixture left to stir at room temperature for $1 \mathrm{~h}$. The solvent was removed and ${ }^{1} \mathrm{H}$ NMR analysis performed on the crude hydrazone product mixture to determine the conversion of glycolaldehyde to erythrose and threose (SI, Figure S27). Column chromatography removed excess diphenyl hydrazine and glycolaldehyde, and chiral HPLC was performed on the mix of threose and erythrose hydrazone products (SI, Figure S26).
In the crude ${ }^{1} \mathrm{H}$ NMR (SI, Figure S27), the triplet at 6.46 ppm corresponds to the hydrazone proton of glycolaldehyde, while the doublets at 6.49 and $6.52 \mathrm{ppm}$ correspond to the hydrazone proton of threose and erythrose, respectively. As these peaks each integrate to one proton, the integrations can be simply used to calculate conversion and threose:erythrose diastereoselectivity. When using deionized water as the solvent for the hydrogel, the conversion of glycolaldehyde into erythrose and threose was ca. $10 \%$, with a diastereoselectivity for threose over erythrose of ca. 2.5:1 (Table 1 and SI, Figures

Table 1. Dimerization of Glycolaldehyde with Benzylglutamine Amide Data on the Hydrogel over $48 \mathrm{~h}$

\begin{tabular}{ccccc} 
entry & solvent & $\begin{array}{c}\text { conversion } \\
(\%)\end{array}$ & $\begin{array}{c}\text { crude NMR dr } \\
\text { erythrose:threose }\end{array}$ & HPLC ee\% \\
\hline 1 & water & 12 & $1.00: 2.77$ & Ery: 2\%; Thr: $2 \%$ \\
2 & water & 10 & $1.00: 2.90$ & Ery: 2\%; Thr: 3\% \\
3 & water & 7 & $1.00: 1.38$ & Ery: 2\%; Thr: 7\% \\
4 & pH 7 & 68 & $1.00: 2.28$ & Ery: 3\%; Thr: 7\% \\
5 & pH 7 & 43 & $1.00: 1.94$ & Ery: 1\%; Thr: 6\% \\
6 & pH 7 & 76 & $1.00: 1.95$ & Ery: 4\%; Thr: $10 \%$ \\
\hline
\end{tabular}

S28-S30). Interestingly, previous work from the Clarke group using a different catalyst exhibited selectivity for erythrose over threose in this reaction, ${ }^{27}$ clearly demonstrating that precise choice of organocatalyst has a profound influence on reaction outcome. Chiral HPLC indicated a small enantiomeric excess for L-threose of ca. $2-7 \%$ and for D-erythrose of ca. 3\%, demonstrating that the chiral organocatalyst is intimately involved in the reaction. Importantly, in the absence of the catalytic gel, no threose or erythrose products were observed.

The reaction was then performed using $\mathrm{pH} 7$ buffer $(0.01 \mathrm{M}$ phosphate buffered saline, PBS) as solvent to form the gel. This allows control of $\mathrm{pH}$ during the reaction-in the unbuffered reaction, the $\mathrm{pH}$ started at ca. 6 and fell slightly during the course of reaction. Pleasingly, the conversion of glycolaldehyde to threose and erythrose increased dramatically to $43-76 \%$ (Table 1 and SI, Figures S31-S33). Once again, in the absence of catalyst, no threose or erythrose was formed. In addition, there was still significant selectivity for threose over erythrose (ca. 2:1). Chiral HPLC again indicated a small ee in favor of L-threose (6.5\%), but a change in the favored enantiomer of erythrose, with small ee in favor of of $\mathrm{L}$ erythrose $(2.5 \%)$.

For purposes of comparison, we performed the dimerization of glycoladehyde reaction using compound 3 as a catalyst in solution, rather than in the gel phase. To achieve this, we added the same amount of compound 3 into water but omitted the heat/cool gel-forming step. Fascinatingly, with conversions of only ca. $5 \%$ either in water or in buffer, and no measurable ee's, the reaction was very significantly less successful than when applying the catalyst in the gel form. This suggests, inline with some other literature reports, ${ }^{11,12,29}$ that the highly organized nanostructure of the gel plays a key role in helping achieve a better reaction outcome. We reason that the nanofibrillar solid-like gel network enhances reactivity and helps ensure effective contact between the organocatalyst and the aqueous-phase reagents.

In summary, hydrogelator $\mathbf{3}$ is catalytically proficient in a prebiotically relevant aldol reaction in the gel phase, and can achieve excellent levels of conversion, along with diastereoselectivity and some enantioselectivity-the first time this has 
been demonstrated for a self-assembled LMWG in an unprotected aqueous-phase prebiotic reaction.

Although the enantioselectivity is relatively low, it is in line with other reports for this reaction. ${ }^{20-24,26-28}$ Furthermore, mechanisms for enantio-enrichment are well-known in the prebiotic literature. ${ }^{46} \mathrm{~A}$ number of these rely on preferential removal of one enantiomer, which is, at least in principle, possible in a chiral gel, where one enantiomer may preferentially interact with the gel network, becoming effectively isolated from the surroundings and hence enriched. ${ }^{47}$ Our reaction workup and characterization methodology did not allow such effects to be observed here, but we suggest it as a potential advantage of gels in prebiotic catalytic systems that is worth further study.

\section{CONCLUSIONS AND FUTURE PERSPECTIVES}

In summary, this investigation into organocatalytic gels used simple prebiotically relevant building blocks and reactions. In this way, we reported a self-assembling glutamine amide derivative 1 , capable of organocatalysis of the reaction between cyclohexanone and 4-nitrobenzaldehyde in "solution", but which could not maintain its gel structure during reaction. In our studies of this simple reaction, we found that compound 1 could itself, on reaction with benzaldehydes, form an effective two-component Schiff base hydrogelator 2 in situ. This versatile, dynamic two-component gel was characterized in some detail, as two-component hydrogels remain rare.

In the ongoing hunt for an effective organocatalyst, we then applied reductive conditions to benzaldehyde-modified Schiff base $2 \mathrm{a}$, to yield secondary amine 3 . This minimal perturbation to the molecular structure yielded a highly effective and wellorganized hydrogelator, active at loadings as low as $0.03 \% \mathrm{wt} /$ vol. Most importantly, this new gel was catalytically proficient for the prebiotically relevant dimerization of glycolaldehyde to give threose and erythrose. The reaction proceeded with good diastereoselectivity, some enantioselectivity, and, when the reaction was performed in buffered conditions, excellent conversions. The reaction using the gel-phase catalyst was much more successful that when the catalyst was used in the solution phase, suggesting that the well-organized nanoscale environment, bringing the catalytic LMWG into intimate contact with the solution-phase reagents, is beneficial for organocatalysis.

This report combines catalyst design with gelator discovery (and vice versa) to generate simple new functional gels with great potential for a range of applications, as well as effective performance in aqueous-phase unprotected prebiotic reaction processes.

In terms of the prebiotic relevance of this general approach, we would highlight the following:

1. LMWGs such as these are based on the self-assembly of prebiotically plausible small molecules.

2. Gelators are preferentially selected and assembled from mixtures of components.

3. The gel catalyzes an unprotected, prebiotically relevant aldol reaction proposed in the synthesis of sugars, in water, with good yield and with diastereo-/enantioselectivity, results which are comparable with other studies.

4. Self-assembly into a gel is required in this case for effective organocatalysis, indicating a potential role for self-assembly in enhancing activity in mixtures.
5. Gels can, at least in principle, provide a unique environment, separated from the bulk yet fully accessible to small molecules that can diffuse in and out-in this way they can be considered like "reaction vessels" or even "primitive cells".

In future work, we intend to extend the scope of gel-phase organocatalysts while continuing to explore minimal systems that are capable of forming self-assembled hydrogels. We hope in the future to demonstrate advantages of compartmentalization within gels. We suggest that self-assembled gels are previously overlooked materials of potential interest in the "systems approach", in which a number of components can collaborate, in terms of both self-assembly and reactivity, to achieve outcomes that may have been of prebiotic importance.

\section{ASSOCIATED CONTENT}

\section{Supporting Information}

The Supporting Information is available free of charge at https://pubs.acs.org/doi/10.1021/jacs.9b13156.

Full characterization of novel compounds, materials and methods, details of analytical techniques, additional spectroscopic and analytical data, and HPLC traces (PDF)

\section{AUTHOR INFORMATION}

\section{Corresponding Authors}

Paul A. Clarke - Department of Chemistry, University of York, York YO10 5DD, U.K.; Email: paul.clarke@york.ac.uk

David K. Smith - Department of Chemistry, University of York, York YO10 5DD, U.K.; (1) orcid.org/0000-0002-9881-2714; Email: david.smith@york.ac.uk

\section{Authors}

Kirsten Hawkins - Department of Chemistry, University of York, York YO10 5DD, U.K.

Anna K. Patterson - Department of Chemistry, University of York, York YO10 5DD, U.K.

Complete contact information is available at:

https://pubs.acs.org/10.1021/jacs.9b13156

\section{Funding}

This research was funded by University of York through an EPSRC DTA award.

\section{Notes}

The authors declare no competing financial interest.

Reference spectroscopic, characterization, reaction, and analytical data can be found at DOI: $10.15124 /$ fa5a7bed-5fda40fc-9bf1-a089136a01e0.

\section{REFERENCES}

(1) (a) Functional Molecular Gels; Escuder, B., Miravet, J. F., Eds.; RSC Publishing: Cambridge, UK, 2013. (b) Molecular Gels: Structure and Dynamics; Weiss, R. G., Ed.; RSC Publishing: Cambridge, UK, 2018.

(2) (a) Weiss, R. G. The Past, Present, and Future of Molecular Gels. J. Am. Chem. Soc. 2014, 136, 7519-7530. (b) Draper, E. R.; Adams, D. J. Low-molecular-weight Gels: The State of the Art. Chem. 2017, 3, 390-410. (c) Amabilino, D. B.; Smith, D. K.; Steed, J. W. Supramolecular Materials. Chem. Soc. Rev. 2017, 46, 2404-2420.

(3) Du, X.; Zhou, J.; Shi, J.; Xu, B. Supramolecular Hydrogelators and Hydrogels: From Soft Matter to Molecular Biomaterials. Chem. Rev. 2015, 115, 13165-13307. 
(4) Okesola, B. O.; Smith, D. K. Applying Low-Molecular Weight Supramolecular Gelators in an Environmental Setting - SelfAssembled Gels as Smart Materials for Pollutant Removal. Chem. Soc. Rev. 2016, 45, 4226-4251.

(5) (a) Hirst, A. R.; Smith, D. K. Two-component Gel-phase Materials - Highly Tunable Self-assembling Systems. Chem. - Eur. J. 2005, 11, 5496-5508. (b) Buerkle, L. E.; Rowan, S. J. Supramolecular Gels formed from Multi-component Low Molecular Weight Species. Chem. Soc. Rev. 2012, 41, 6089-6102. (c) Raeburn, J.; Adams, D. J. Multicomponent Low Molecular Weight Gelators. Chem. Commun. 2015, 51, 5170-5180. (d) Draper, E. R.; Adams, D. J. How Should Multicomponent Supramolecular Gels be Characterised? Chem. Soc. Rev. 2018, 47, 3395-3405.

(6) (a) Hanabusa, K.; Miki, T.; Taguchi, Y.; Koyama, T.; Shirai, H. Two-component Small Molecule Gelling Agents. J. Chem. Soc., Chem. Commun. 1993, 1382-1384. (b) Partridge, K. S.; Smith, D. K.; Dykes, G. M.; McGrail, P. T. Supramolecular Dendritic Two-Component Gel. Chem. Commun. 2001, 319-320. (c) Babu, P.; Sangeetha, N. M.; VijayKumar, P.; Maitra, U.; Rissanen, K.; Raju, A. R. Pyrene-Derived Novel One and Two-Component Organgelators. Chem. - Eur. J. 2003, 9, 1922-1932. (d) Basit, H.; Pal, A.; Sen, S.; Bhattacharya, S. TwoComponent Hydrogels Comprising Fatty Acids and Amines: Structure, Properties and Application as a Template for the Synthesis of Metal Nanoparticles. Chem. - Eur. J. 2008, 14, 6534-6545. (e) Rao, K. V.; Jayaramulu, K.; Maji, T. K.; George, S. J. Supramolecular Hydrogels and High-Aspect-Ratio Nanofibers through ChargeTransfer-Induced Alternate Coassembly. Angew. Chem., Int. Ed. 2010, 49, 4218-4222. (f) Xia, Q.; Mao, Y.; Wu, J.; Shu, T.; Yi, T. Two-Component Organogel for Visually Detecting Nitrite Anion. J. Mater. Chem. C 2014, 2, 1854-1861. (g) Su, T.; Hong, K. H.; Zhang, W.; Li, F.; Li, Q.; Yu, F.; Luo, G.; Gao, H.; He, Y.-P. Scaleable TwoComponent Gelator from Phthalic Acid Derivatives and Primary Alkyl Amines: Acid-Base Interaction in the Cooperative Assembly. Soft Matter 2017, 13, 4066-4073. (h) Roy, R.; Adalder, T. K.; Dastidar, P. Supramolecular Gels Derived from the Salts of Variously Substituted Phenylacetic Acid and Dicyclohexylamine: Design, Synthesis, Structures, and Dye Adsorption. Chem. - Asian J. 2018, 13, 552-559.

(7) (a) de Hatten, X.; Bell, N.; Yufa, N.; Christmann, G.; Nitschke, J. R. A Dynamic Covalent, Luminescent Metallopolymer that Undergoes Sol-to-Gel Transition on Temperature Rise. J. Am. Chem. Soc. 2011, 133, 3158-3164. (b) Minkenberg, C. B.; Hendriksen, W. E.; Li, F.; Mendes, E.; Eelkema, R.; van Esch, J. H. Dynamic Covalent Assembly of Stimuli Responsive Vesicle Gels. Chem. Commun. 2012, 48, 9837-9839. (c) Luo, W.; Zhu, Y.; Zhang, J.; He, J.; Chi, Z.; Miller, P. W.; Chen, L.; Su, C.-Y. A Dynamic Covalent Gel as a Luminescent Sensor. Chem. Commun. 2014, 50, 11942-11945. (d) Khalily, M. A.; Goktas, M.; Guler, M. O. Tuning Viscoelastic Properties of Supramolecular Peptide Gels via Dynamic Covalent Crosslinking. Org. Biomol. Chem. 2015, 13, 1983-1987.

(8) (a) Zhang, J.-Y.; Zeng, L.-H.; Feng, J. Dynamic Covalent Gels Assembled From Small Molecules: From Discrete Gelators to Dynamic Covalent Polymers. Chin. Chem. Lett. 2017, 28, 168-183. (b) Zhang, Z.; He, C.; Chen, X. Hydrogels based on pH-responsive reversible carbon-nitrogen double-bond linkages for biomedical applications. Mater. Chem. Front. 2018, 2, 1765-1778.

(9) (a) Zhang, J.-Y.; Zeng, L.-H.; Feng, J. Dynamic Covalent Gels Assembled From Small Molecules: From Discrete Gelators to Dynamic Covalent Polymers. Chin. Chem. Lett. 2017, 28, 168-183. (b) Sreenivasachary, N.; Lehn, J.-M. Gelation-Driven Component Selection in the Generation of Constitutional Dynamic Hydrogels Based on Guanine-Quartet Formation. Proc. Natl. Acad. Sci. U. S. A. 2005, 102, 5938-5943. (c) Boekhoven, J.; Poolman, J. M.; Maity, C.; Li, F.; van der Mee, L.; Minkenberg, C. B.; Mendes, E.; van Esch, J. H.; Eelkema, R. Catalytic Control over Supramolecular Gel Formation. Nat. Chem. 2013, 5, 433-437. (d) Foster, J. S.; Żurek, J. M.; Almeida, N. M. S.; Hendriksen, W. E.; le Sage, V. A. A.; Lakshminarayanan, V.; Thompson, A. L.; Banerjee, R.; Eelkema, R.; Mulvana, H.; Paterson, M. J.; van Esch, J. H.; Lloyd, G. O. Gelation Landscape Engineering Using a Multi-Reaction Supramolecular
Hydrogelator System. J. Am. Chem. Soc. 2015, 137, 14236-14239. (e) Beckers, S. J.; Parkinson, S.; Wheeldon, E.; Smith, D. K. In situ Aldehyde-Modification of Self-Assembled Acyl Hydrazide Hydrogels and Dynamic Component Selection from Complex Aldehyde Mixtures. Chem. Commun. 2019, 55, 1947-1950.

(10) (a) Escuder, B.; Rodriguez-Llansola, F.; Miravet, J. F. Supramolecular Gels as Active Media for Organic Reactions and Catalysis. New J. Chem. 2010, 34, 1044-1054. (b) Fang, W.; Zhang, Y.; Wu, J.; Liu, C.; Zhu, H.; Tu, T. Recent Advances in Supramolecular Gels and Catalysis. Chem. - Asian J. 2018, 13, 712729. (c) Dawn, A. Supramolecular Gel as the Template for Catalysis, Inorganic Superstructure, and Pharmaceutical Crystallization. Int. J. Mol. Sci. 2019, 20, 781.

(11) (a) Guler, M. O.; Stupp, S. I. A Self-Assembled Nanofiber Catalyst for Ester Hydrolysis. J. Am. Chem. Soc. 2007, 129, 1208212083. (b) Bhat, S.; Maitra, U. Hydrogels as reaction vessels: Acenaphthylene dimerization in hydrogels derived from bile acid analogues. Molecules 2007, 12, 2181-2189. (c) Dawn, A.; Fujita, N.; Haraguchi, S.; Sada, K.; Shinkai, S. An organogel system can control the stereochemical course of anthracene photodimerization. Chem. Commun. 2009, 2100-2102. (d) Samai, S.; Ghosh, P.; Biradha, K. Does Crystal or Gel Matter to Stereochemistry of a Reaction? Silver Complexation-Promoted Solid-State $[2+2]$ Reaction of an Unsymmetrical Olefin. Chem. Commun. 2013, 49, 4181-4183. (e) Bachl, J.; Hohenleutner, A.; Dhar, B. B.; Cativiela, C.; Maitra, U.; König, B.; Díaz, D. D. Organophotocatalysis in nanostructured soft gel materials as tunable reaction vessels: comparison with homogeneous and micellar solutions. J. Mater. Chem. A 2013, 1, 4577-4588. (f) Zhang, C.; Xue, X.; Luo, Q.; Li, Y.; Yang, K.; Zhuang, X.; Jiang, Y.; Zhang, J.; Liu, J.; Zou, G.; Liang, X.-J. Self-Assembled Peptide Nanofibers Designed as Biological Enzymes for Catalyzing Ester Hydrolysis. ACS Nano 2014, 8, 11715-11723. (g) Rufo, C. M.; Moroz, Y. S.; Moroz, O. V.; Stöhr, J.; Smith, T. A.; Hu, X.; DeGrado, W. F.; Korendov ych, I. V. Short Peptides Self-Assemble to Produce Catalytic Amyloids. Nat. Chem. 2014, 6, 303-309. (h) Belieres, M.; Chouini-Lalanne, N.; Dejugnat, C. Synthesis, Self-Assembly, and Catalytic Activity of Histidine-Based Structured Lipopeptides for Hydrolysis Reactions in water. RSC Adv. 2015, 5, 35830-35842. (i) Neumann, L. N.; Baker, M. B.; Leenders, C. M. A.; Voets, I. K.; Lafleur, R. P. M.; Palmans, A. R. A.; Meijer, E. W. Supramolecular Polymers for Organocatalysis in Water. Org. Biomol. Chem. 2015, 13, 7711-7719. (j) Zhang, C.; Shafi, R.; Lampel, A.; MacPherson, D.; Pappas, C. G.; Narang, V.; Wang, T.; Maldarelli, C.; Ulijn, R. V. Switchable Hydrolase Based on Reversible Formation of Supramolecular Catalytic Site Using a Self-Assembling Peptide. Angew. Chem., Int. Ed. 2017, 56, 14511-14515. (k) Gayen, K.; Basu, K.; Bairagi, D.; Castelletto, V.; Hamley, I. W.; Banerjee, A. Amino-acidbased metallo-hydrogel that acts like an esterase. ACS Appl. Bio Mater. 2018, 1, 1717-1724. (1) Haring, M.; Abramov, A.; Okumura, K.; Ghosh, I.; Konig, B.; Yanai, N.; Kimizuka, N.; Diaz Diaz, D. Airsensitive photoredox catalysis performed under aerobic conditions in gel networks. J. Org. Chem. 2018, 83, 7928-7938.

(12) (a) Rodriguez-Llansola, F.; Escuder, B.; Miravet, J. F. Switchable Performance of an 1-Proline Derived Basic Catalyst Controlled by Supramolecular Gelation. J. Am. Chem. Soc. 2009, 131, 11478-11484. (b) Rodriguez-Llansola, F.; Miravet, J. F.; Escuder, B. A Supramolecular Hydrogel as a Reusable Heterogeneous Catalyst for the Direct Aldol Reaction. Chem. Commun. 2009, 73037305. (c) Rodriguez-Llansola, F.; Miravet, J. F.; Escuder, B. Supramolecular Catalysis with Extended Aggregates and Gels: Inversion of Stereoselectivity Caused by Self-Assembly. Chem. - Eur. J. 2010, 16, 8480-8486. (d) Diaz-Oltra, S.; Berdugo, C.; Miravet, J. F.; Escuder, B. Study of the Effect of Polymorphism on the SelfAssembly and Catalytic Performance of an 1-Proline Based Molecular Hydrogelator. New J. Chem. 2015, 39, 3785-3791.

(13) Singh, N.; Zhang, K.; Angulo-Pachon, C. A.; Mendes, E.; van Esch, J. H.; Escuder, B. Tandem Reactions in Self-sorted Catalytic Molecular Hydrogels. Chem. Sci. 2016, 7, 5568-5572. 
(14) (a) Cleaves, H. J. Prebiotic Chemistry: What We Know, What We Don't. Evolution: Educ. And Outreach 2012, 5, 342-360. (b) Islam, S.; Powner, M. W. Prebiotic Systems Chemistry: Complexity. Overcoming Clutter. Chem. 2017, 2, 470-501. (c) Sutherland, J. D. Opinion: Studies on the Origin of Life - The End of the Beginning. Nat. Rev. Chem. 2017, 1, 0012. (d) Kitadai, N.; Maruyama, S. Origins of Building Blocks of Life: A Review. Geosci. Front. 2018, 9, $1117-1153$.

(15) (a) Barbas, C. F. Organocatalysis Lost: Modern Chemistry, Ancient Chemistry and an Unseen Biosynthetic Apparatus. Angew. Chem., Int. Ed. 2008, 47, 42-47.

(16) (a) Butlerow, A. Formation Synthetique d'une Substance Sucrée. Compt. Rend. Acad. Sci. 1861, 53, 145-147. (b) Breslow, R. On the Mechanism of the Formose Reaction. Tetrahedron Lett. 1959, 1, 22-26.

(17) (a) Ritson, D.; Sutherland, J. D. Prebiotic Synthesis of Simple Sugars by Photoredox Systems Chemistry. Nat. Chem. 2012, 4, 895899. (b) Ritson, D. J.; Sutherland, J. D. Synthesis of Aldehydic Ribonucleotide and Amino Acid Precursors by Photoredox Chemistry. Angew. Chem., Int. Ed. 2013, 52, 5845-5847.

(18) (a) Eder, U.; Sauer, G.; Wiechert, R. New Type of Asymmetric Cyclization to Optically Active Steroid CD Partial Structures. Angew. Chem., Int. Ed. Engl. 1971, 10, 496-497. (b) Hajos, Z. G.; Parrish, D. R. Asymmetric Synthesis of Bicyclic Intermediates of Natural Product Chemistry. J. Org. Chem. 1974, 39, 1615-1621.

(19) (a) List, B.; Lerner, R. A.; Barbas, C. F. Proline-Catalysed Direct Asymmetric Aldol Reactions. J. Am. Chem. Soc. 2000, 122, 2395-2396. (b) Sakthivel, K.; Notz, W.; Bui, T.; Barbas, C. F. Amino Acid Catalyzed Diret Asymmetric Aldol Reactions: A Bioorganic Approach to Catalytic Asymmetric Carbon-Carbon Bond-Forming Reactions. J. Am. Chem. Soc. 2001, 123, 5260-5267. (c) Notz, W.; Tanaka, F.; Barbas, C. F. Enamine-Based Organocatalysis with Proline and Diamines: The Development of Direct Catalytic Asymmetric Aldol, Mannich, Michael, and Diels-Alder Reactions. Acc. Chem. Res. 2004, 37, 580-591.

(20) Pizarello, S.; Weber, A. L. Prebiotic Amino Acids as Asymmetric Catalysts. Science 2004, 303, 1151.

(21) (a) Breslow, R.; Cheng, Z.-L. l-Amino Acids Catalyze the Formation of an Excess of d-Glyceraldehyde, and thus of Other dSugars Under Credible Prebiotic Conditions. Proc. Natl. Acad. Sci. U. S. A. 2010, 107, 5723-5725. (b) Breslow, R.; Ramalingam, V.; Appayee, C. Catalysis of glyceraldehyde synthesis by primary or secondary amino acids under prebiotic conditions as a function of $\mathrm{pH}$. Origins Life Evol. Biospheres 2013, 43, 323-329.

(22) Weber, A. L.; Pizzarello, S. The Peptide-Catalyzed Stereospecific Synthesis of Tetroses: A Possible Model for Prebiotic Molecular Evolution. Proc. Natl. Acad. Sci. U. S. A. 2006, 103, 1271312717.

(23) (a) Kofoed, J.; Machuqueiro, M.; Reymond, J.-L.; Darbre, T. Zinc-Proline Catalyzed Pathway for the Formation of Sugars. Chem. Commun. 2004, 1540-1541. (b) Kofoed, J.; Reymond, J.-L.; Darbre, T. Prebiotic Carbohydrate Synthesis: Zinc-Proline Catalyzes Direct Aqueous Aldol Reactions of $\alpha$-Hydroxy Aldehydes and Ketones. Org. Biomol. Chem. 2005, 3, 1850-1855.

(24) Mase, N.; Nakai, Y.; Ohara, N.; Yoda, H.; Takabe, K.; Tanaka, F.; Barbas, C. F. Organocatalytic Direct Asymmetric Aldol Reactions in Water. J. Am. Chem. Soc. 2006, 128, 734-735.

(25) Brogan, A. P.; Dickerson, T. J.; Janda, K. D. Enamine-Based Aldol Organocatalysis in Water: Are They Really "All Wet"? Angew. Chem., Int. Ed. 2006, 45, 8100-8102.

(26) Burroughs, L.; Vale, M. E.; Gilks, J. A. R.; Forintos, H.; Hayes, C. J.; Clarke, P. A. Efficient Asymmetric Organocatalytic Formation of Erythrose and Threose under Aqueous Conditions. Chem. Commun. 2010, 46, 4776-4778.

(27) Burroughs, L.; Clarke, P. A.; Forintos, H.; Gilks, J. A. R.; Hayes, C. J.; Vale, M. E.; Wade, W.; Zbytniewski, M. Asymmetric Organocatalytic Formation of Protected and Unprotected Tetroses under Potentially Prebiotic Conditions. Org. Biomol. Chem. 2012, 10, $1565-1570$.
(28) Steer, A. M.; Bia, N.; Smith, D. K.; Clarke, P. A. Prebiotic Synthesis of 2-Deoxy-d-Ribose from Interstellar Building Blocks Promoted by Amino Ester or Amino Nitriles. Chem. Commun. 2017, 53, 10362-10365.

(29) Tena-Solsona, M.; Nanda, J.; Diaz-Oltra, S.; Chotera, A.; Ashkenasy, G.; Escuder, B. Emergent Catalytic Behaviour of SelfAssembled Low Molecular Weight Peptide-Based Aggregates and Hydrogels. Chem. - Eur. J. 2016, 22, 6687-6694.

(30) Pollack, G. H. Cells, Gels and the Engines of Life: A new, unifying approach to cell function; Oxford University Press: Oxford, 2001.

(31) Trevors, J. T.; Pollack, G. H. Hypothesis: The Origin of Life in a Hydrogel Environment. Prog. Biophys. Mol. Biol. 2005, 89, 1-8.

(32) (a) Ruiz-Mirazo, K.; Briones, C.; de la Escosura, A. Prebiotic Systems Chemistry: New Perspectives for the Origins of Life. Chem. Rev. 2014, 114, 285-366. (b) Islam, S.; Powner, M. W. Prebiotic Systems Chemistry: Complexity Overcoming Clutter. Chem. 2017, 2, $470-501$.

(33) Steer, A. M. Studies on the Prebiotic Origin of 2-Deoxy-D-ribose. Ph.D. Thesis, University of York, 2017.

(34) (a) Wang, G. T.; Lin, J. B.; Jiang, X. K.; Li, Z. T. CholesterolAppended Aromatic Imine Organogelators: A Case Study of GelationDriven Component Selection. Langmuir 2009, 25, 8414-8418. (b) Jin, Q. X.; Zhang, L.; Zhu, X. F.; Duan, P. F.; Liu, M. H. Amphiphilic Schiff Base Organogels: Metal-Ion Mediated Chiral Twists and Chiral Recognition. Chem. - Eur. J. 2012, 18, 4916-4922. (c) Lv, K.; Qin, L.; Wang, X. F.; Zhang, L.; Liu, M. H. A Chiroptical Switch Based on Supramolecular Chirality Transfer Through Alkyl Chain Entanglement and Dynamic Covalent Bonding. Phys. Chem. Chem. Phys. 2013, 15, 20197-20202. (d) Bunzen, H.; Nonappa; Kalenius, E.; Hietala, S.; Kolehmainen, E. Subcomponent SelfAssembly: A Quick Way to New Metallogels. Chem. - Eur. J. 2013, 19, 12978-12981. (e) Zang, L. B.; Shang, H. X.; Wei, D. Y.; Jiang, S. M. A Multi-Stimuli-Responsive Organogel Based on Salicylidene Schiff Base. Sens. Actuators, B 2013, 185, 389-397. (f) Hu, L.; Zhang, Y.; Ramström, O. Gelation-Driven Systemic Resolution: in situ Generation and Self-Selection of an Organogelator. Sci. Rep. 2015, 5, 11065. (g) Panja, A.; Ghosh, K. Azo and Imine Functionalized 2Naphthols: Promising Supramolecular Gelators for Selective Detection of $\mathrm{Fe}^{3+}$ and $\mathrm{Cu}^{2+}$, Reactive Oxygen Species and Halides. Mater. Chem. Front. 2018, 2, 1866-1875. (h) Panja, A.; Ghosh, K. 4Hydroxybenzaldehyde Derived Schiff Based Gelators: Case of the Sustainability or Rupturing of Imine Bonds Towards the Selective Sensing of $\mathrm{Ag}^{+}$and $\mathrm{Hg}^{2+}$ Ions via Sol-Gel Methodology. New J. Chem. 2019, 43, 5139-5149. (i) Mondal, S.; Bairi, P.; Das, S.; Nandi, A. K. Phase Selective Organogel from an Imine Based Gelator for use in Oil Spill Recovery. J. Mater. Chem. A 2019, 7, 381-392.

(35) Gronwald, O.; Shinkai, S. Sugar-Integrated Gelators of Organic Solvents. Chem. - Eur. J. 2001, 7, 4328-4334.

(36) Escuder, B.; Llusar, M.; Miravet, J. F. Insight on the NMR Study of Supramolecular Gels and Its Application to Monitor Molecular Recognition on Self-Assembled Fibers. J. Org. Chem. 2006, 71, 7747-7752.

(37) Draper, E. R.; Wallace, M.; Schweins, R.; Poole, R. J.; Adams, D. J. Nonlinear Effects in Multicomponent Supramolecular Hydrogels. Langmuir 2017, 33, 2387-2395.

(38) Adams, D. J.; Butler, M. F.; Frith, W. J.; Kirkland, M.; Mullen, L.; Sanderson, P. A New Method for Maintaining Homogeneity During Liquid-Hydrogel Transitions Using Low Molecular Weight Hydrogelators. Soft Matter 2009, 5, 1856-1862.

(39) Gawronski, J.; Grajewski, J. The Significance of Induced Circular Dichroism. Org. Lett. 2003, 5, 3301-3303.

(40) (a) Mears, L. L. E.; Draper, E. R.; Castilla, A. M.; Su, H.; Zhuola; Dietrich, B.; Nolan, M. C.; Smith, G. N.; Doutch, J.; Rogers, S.; Akhtar, R.; Cui, H.; Adams, D. J. Drying Affects the Fiber Network in Low Molecular Weight Hydrogels. Biomacromolecules 2017, 18, 3531-3540. (b) Adams, D. J. Does Drying Affect Gel Networks. Gels 2018, $4,32$.

(41) Li, J.; Geng, L.; Wang, G.; Chu, H.; Wei, H. Self-Healable Gels for Use in Wearable Devices. Chem. Mater. 2017, 29, 8932-8952. 
(42) (a) Menor-Salván, C.; Marin-Yaseli, M. R. Prebiotic Chemistry in Eutectic Solutions at the Water-Ice Matrix. Chem. Soc. Rev. 2012, 41, 5404-5415. (b) Handbook of Astrobiology, Kolb, V. M., Ed.; CRC Press, Taylor and Francis, Boca Raton, FL, 2019; p 333.

(43) (a) Sreenivasachary, N.; Lehn, L.-M. Gelation-Driven Component Selection in the Generation of Constitutional Dynamic Hydrogels Based on Guanine Quartet Formation. Proc. Natl. Acad. Sci. U. S. A. 2005, 102, 5938-5943. (b) Hirst, A. R.; Miravet, J. F.; Escuder, B.; Noirez, L.; Castelletto, V.; Hamley, I. W.; Smith, D. K. Self-Assembly of Two-Component Gels: Stoichiometric Control and Component Selection. Chem. - Eur. J. 2009, 15, 372-379. (c) Smith, M. M.; Edwards, W.; Smith, D. K. Self-Organisation Effects in Dynamic Nanoscale Gels Self-Assembled from Simple Mixtures of Commercially Available Molecular-Scale Components. Chem. Sci. 2013, 4, 671-676. (d) Beckers, S. J.; Parkinson, S.; Wheeldon, E.; Smith, D. K. In Situ Aldehyde-Modification of Self-Assembled Acyl Hydrazide Hydrogels and Dynamic Component Selection from a Complex Aldehyde Mixture. Chem. Commun. 2019, 55, 1947-1950.

(44) Islam, S.; Bučar, D. K.; Powner, M. W. Prebiotic Selection and Assembly of Proteinogenic Amino Acids and Natural Nucleotides from Complex Mixtures. Nat. Chem. 2017, 9, 584-589.

(45) (a) Huber, C.; Wächtershäuser, G. Primordial Reductive Amination Revisited. Tetrahedron Lett. 2003, 44, 1695-1697. (b) Wang, W.; Li, Q.; Yang, B.; Liu, X.; Yang, Y.; Su, W. Photocatalytic Reversible Amination of a-Keto Acids on a $\mathrm{ZnS}$ Surface: Implications for the Prebiotic Metabolism. Chem. Commun. 2012, 48, 2146-2148. (c) Gounaris, Y.; Litinas, C.; Evgenidou, E. A Possible Prebiotic Function of Cytosine as Amino Acid Synthesizer. Hypothesis 2014, 12, No. e5. Barge, L. M.; Flores, E.; Baum, M. M.; van der Velde, D. G.; Russell, M. J. Redox and pH Gradients Drive Amino Acid Synthesis in Iron Oxyhydroxide Mineral Systems. Proc. Natl. Acad. Sci. U. S. A. 2019, 116, 4828-4833.

(46) Blackmond, D. G. The Origin of Biological Homochirality. Philos. Trans. R. Soc., B 2011, 366, 2878-2884.

(47) Edwards, W.; Smith, D. K. Enantioselective Component Selection in Multicomponent Supramolecular Gels. J. Am. Chem. Soc. 2014, 136, 1116-1124. 